Portland State University

PDXScholar

Civil and Environmental Engineering Faculty

Publications and Presentations

Civil and Environmental Engineering

$5-2009$

\title{
Vertical Boil Propagation from a Submerged Estuarine Sill
}

C. Chris Chickadel

University of Washington - Seattle Campus

Alexander R. Horner-Devine

University of Washington - Seattle Campus

Stefan A. Talke

Portland State University, talke@pdx.edu

Andrew T. Jessup

University of Washington - Seattle Campus

Follow this and additional works at: https://pdxscholar.library.pdx.edu/cengin_fac

Part of the Civil Engineering Commons, and the Environmental Engineering Commons Let us know how access to this document benefits you.

Citation Details

Chickadel, C. C., A. R. Horner-Devine, S. A. Talke, and A. T. Jessup (2009), Vertical boil propagation from a submerged estuarine sill, Geophys. Res. Lett., 36, L10601, doi:10.1029/2009GL037278.

This Article is brought to you for free and open access. It has been accepted for inclusion in Civil and Environmental Engineering Faculty Publications and Presentations by an authorized administrator of PDXScholar. Please contact us if we can make this document more accessible: pdxscholar@pdx.edu. 


\title{
Vertical boil propagation from a submerged estuarine sill
}

\author{
C. Chris Chickadel, ${ }^{1}$ Alexander R. Horner-Devine, ${ }^{2}$ \\ Stefan A. Talke, ${ }^{2}$ and Andrew T. Jessup ${ }^{1}$ \\ Received 13 January 2009; revised 1 April 2009; accepted 17 April 2009; published 20 May 2009.
}

[1] Surface disruptions by boils during strong tidal flows over a rocky sill were observed in thermal infrared imagery collected at the Snohomish River estuary in Washington State. Locations of boil disruptions and boil diameters at the surface were quantified and are used to test an idealized model of vertical boil propagation. The model is developed as a two-dimensional approximation of a three-dimensional vortex loop, and boil vorticity is derived from the flow shear over the sill. Predictions of boil disruption locations were determined from the modeled vertical velocity, the sill depth, and the over-sill velocity. Predictions by the vertical velocity model agree well with measured locations (rms difference $3.0 \mathrm{~m}$ ) and improve by using measured velocity and shear (rms difference $1.8 \mathrm{~m}$ ). In comparison, a boil-surfacing model derived from laboratory turbulent mixed-layer wakes agrees with the measurements only when stratification is insignificant. Citation: Chickadel, C. C., A. R. Horner-Devine, S. A. Talke, and A. T. Jessup (2009), Vertical boil propagation from a submerged estuarine sill, Geophys. Res. Lett., 36, L10601, doi:10.1029/2009GL037278.

\section{Introduction}

[2] Bedforms, abrupt bathymetry gradients, and obstructions are common to rivers and estuaries and serve as generation points for meter-scale coherent structures often called "boils". Boils are a key mechanism for transport and redistribution of momentum, temperature, nutrients, and sediment. Characterization of their kinematics is important for understanding and modeling of estuarine physical, chemical, biological, and geomorphologic processes [Matthes, 1947].

[3] The generation of boils in laboratory experiments is described as the interaction of span-wise vorticity resulting from flow separation over bottom topography or hydrodynamic instability, which is deformed into hairpin-shaped loops of spanwise vorticity [Müller and Gyr, 1986]. Loops with downstream bends will vertically self-advect and erupt at the surface [Nezu and Nakagawa, 1993; Müller and Gyr, 1986]. Detailed field investigations [Venditti and Bauer, 2005] and models of laboratory flow over dunes [Best, 2005a] reveal flow separation at dune crests where Kelvin-Helmoltz instabilities are generated in a mixed-layer wake. In these experiments, boil-type features appear to be generated at the downstream point of flow reattachment at

\footnotetext{
${ }^{1}$ Applied Physics Laboratory, University of Washington, Seattle, Washington, USA

${ }^{2}$ Civil and Environmental Engineering, University of Washington, Seattle, Washington, USA.

Copyright 2009 by the American Geophysical Union. 0094-8276/09/2009GL037278
}

the bed. Best [2005b] suggests that observations of surface boils in rivers are consistent with the interaction of a hairpin vortex with the water surface. Large-eddy simulation (LES) numerical models used to examine the structure of the turbulent wake from a dune [Yue et al., 2005, 2006] and direct numerical simulations [Tsai, 1998] showed hairpinshaped vortical motions were generated within the shear layer, consistent with laboratory models. Yue et al. [2005, 2006] and Tsai [1998] extended their models of vorticity to the free surface and found that the vortices create localized upwelling regions as they impinge on the water surface.

[4] The lack of detailed in situ measurements of boils makes it difficult to verify laboratory observations and numerical models of boil generation and development. However, descriptive field observations of the surface signature of boils give some insight into boil kinematics [Coleman, 1969; Jackson, 1976; Kostaschuk and Church, 1993; Babakaiff and Hickin, 1996; Best, 2005b]. The boil eruption sequence at the surface begins as a vigorous incipient upwelling with significant surface deflection. The upwelling patch spreads to a diameter of meters and become less energetic, persisting for tens of seconds. Little vertical vorticity is associated with the main boil eruption patch, though secondary vortices are often seen on the outer edges. Thus laboratory, numerical, and visual observations support a hairpin vortex loop model of boil kinematics.

[5] The difficulty of quantifying boils in the field motivates our use of remote sensing of surface boil parameters to test the hairpin vortex loop model. In July 2006 an experiment to characterize coherent turbulent structures in tidally dominated river flow (the COHerent STructures in Rivers and Estuaries eXperiment, or COHSTREX) was conducted in the Snohomish River estuary in Washington State. Calibrated thermal (longwave infrared) remotely sensed imagery and in situ instruments were used to observe flow over a $4 \mathrm{~m}$ high submerged rocky sill. Because the Snohomish River is a salt wedge estuary [MacDonald and Horner-Devine, 2008], large vertical and horizontal temperature gradients were present due to intrusion of cold saltwater beneath the river water. Infrared remote sensing provided synoptic spatial and temporal sampling of the flow field and revealed significant activity of meter-sized boils generated during periods of strong tidal flow over the sill (Figure 1a). These boils appeared to be transporting colder underlying seawater to the surface, making them visible in thermal imagery. The spatial and temporal patterns of boils observed with infrared imagery, and the fixed location and geometry of the submerged sill, make this a unique data set to investigate boil kinematics. Here, we use the infrared imagery from an ebb tide to determine boil size and the eruption point of boils downstream of the sill. Vertical boil velocity is inferred from the flow depth and horizontal advection speed. These data are 

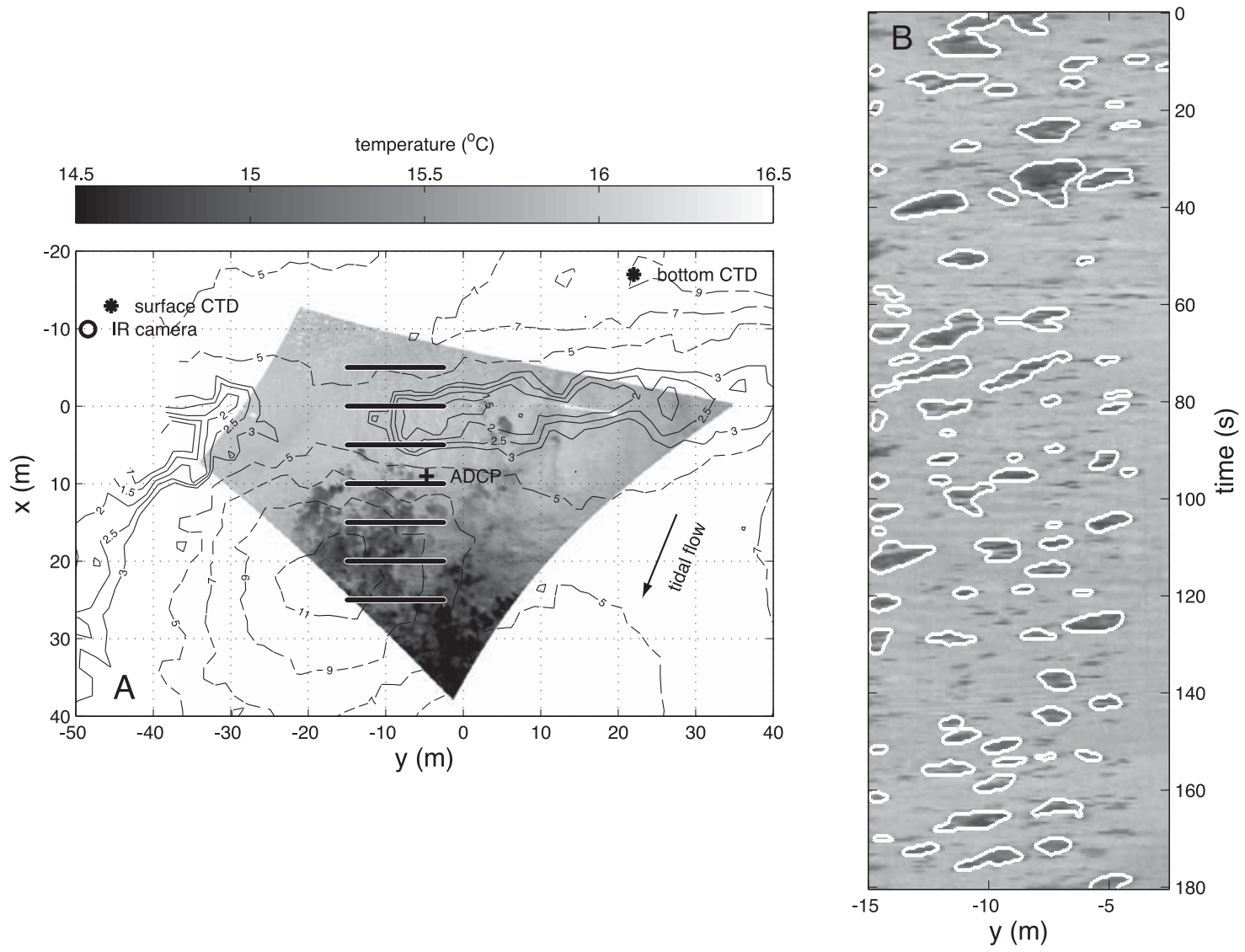

Figure 1. (a) An example rectified thermal image of tidal flow over the submerged sill showing cool (darker) boil eruptions downstream of the sill crest. Intertidal depth contours in meters (solid lines), subtidal contours (dashed lines), and instrument locations are shown. Thermal video was sub-sampled from the seven parallel arrays (horizontal lines) downstream of the shallowest part of the sill, where boils activity was greatest. (b) An example timestack plot of IR imagery from the array at $\mathrm{y}=-10 \mathrm{~m}$ shows cool boils autonomously identified (white outlines, see section 3).

used to test a model of boil upwelling velocity based on the vertical propagation of a vortex dipole.

\section{Vertical Boil Velocity Model}

[6] We propose a theoretical boil vertical propagation model based on the assumption that the boils observed in COHSTREX were surface eruptions of hairpin vortices. Without evidence to the contrary, we assume that the boil vorticity loops originate at the abrupt bathymetry change at the crest of the submerged rocky sill. Boils generated there are carried horizontally with the river flow and upwards by self-advection due to the vortex loop self-interaction.

[7] The vertical velocity of a boil, $w_{b}$, due to a threedimensional vorticity loop is simplified as the self-advection of an irrotational vortex dipole in the vertical plane [Batchelor, 1967],

$$
w_{b}=\frac{\Gamma_{b}}{2 \pi d},
$$

where $\Gamma_{b}$ is the boil circulation and $d$ is the distance between vortex pair centers. As shown later, the lateral scale of the boils observed on the surface is similar to the depth of the shear layer, so we approximate $d$ and the vortex diameter as the observable surface boil diameter. Circulation for each vortex in the pair is the product of the vorticity, $\omega$, and the vortex cross-section, $d A$,

$$
\Gamma_{b}=\int \omega d A \approx \omega \pi\left(\frac{d}{2}\right)^{2}
$$

As is discussed in section 3, the sill extends over $2 / 3$ of the water column during the observation period. Thus, the vorticity generated by shear at the sill crest is expected to be significantly greater than vorticity carried into this region from the bottom boundary layer upstream of the sill. We assume, therefore, that $\omega$ is generated at the sill crest, i.e., $\omega \approx$ $\frac{\partial u}{\partial z}$, where $u$ is the streamwise velocity, $z$ is the vertical coordinate, and vertical velocity is assumed negligible. We further simplify and express the shear in terms of bulk parameters, $\omega \approx \frac{U}{h}$, where $U$ is the surface velocity over the sill and $h$ is the depth of water over the sill crest. Hence, the predicted distance downstream from the sill to the eruption point at the surface, $L_{1}$, is to first order

$$
L_{1}=\frac{U h}{w_{b}},
$$

assuming $w_{b}$ is constant and the boil travels downstream at constant velocity $U$. Combining equation (1) through 
equation (3) results in a simple expression for the downstream boil eruption point,

$$
L_{1}=\frac{8 h^{2}}{d},
$$

which depends only on boil diameter and the sill depth, as $U$ has been eliminated due to kinematics defined in terms of streamwise velocity. Following a similar derivation, but substituting the measured $U$ into equation (3) and the measured vertical gradient in streamwise velocity $U_{z}$ for $\omega$ in equation (2), $L$ can also be estimated as

$$
L_{2}=\frac{2 h U}{d U_{z}} .
$$

\section{Infrared Boil Characterization and Model Testing}

[8] Thermal image data from an ebb tide during COHSTREX on 14 July 2006 was analyzed for boil characteristics, including spanwise boil diameter and horizontal distance downstream of the sill. Imagery of surface flow was collected at a rate of $2 \mathrm{~Hz}$ from a calibrated thermal infrared camera elevated on an extendable hydraulic lift $\sim 35 \mathrm{~m}$ above the river surface. The lift was floated on a barge positioned in a shallow bypass away from the main channel flow, thus allowing for detailed imagery while effectively limiting influence on the observed flow over the sill. The imagery was corrected for radial lens distortion and rectified to realworld coordinates as plan-view images at the measured tide level [Holland et al., 1997]. Image geometry was solved for on a frame-by-frame basis to remove the camera sway due to barge motion. Tidal-flow depth and vertical density stratification were characterized from conductivity, temperature and depth (CTD) measurements from bottom and surface moorings located just upstream of the study site as noted in Figure 1a. Velocity profiles downstream of the sill were collected from a bottom-mounted acoustic doppler current profiler (ADCP) located adjacent to the sill crest. During the analysis period the depth over the sill decreased from $1.5 \mathrm{~m}$ to $1 \mathrm{~m}$ and the surface streamwise flow was $\sim 0.5 \mathrm{~m} \mathrm{~s}^{-1}$.

[9] Boils were identified in an automated procedure operating on a reduced image data set to simplify analysis. Each rectified image frame was sub-sampled in seven arrays parallel to the sill axis (Figure 1a). The arrays are aligned roughly perpendicular to the dominant ebb flow direction and positioned over the east section of the sill. This location was visually determined to be in the path of the most apparent boil activity and is associated with the shallowest part of the sill.

[10] Cold boils advecting past each array were identified against the warmer surrounding surface water by a simple thresholding technique in the thermal time series (Figure 1b). First, the spatial and temporal mean from the data from each array are removed. Next, apparent temperature fluctuations due to short surface waves, roughness, and image noise are reduced to less than $1 \%$ of their spectral variance by Fourier domain low-pass filtering of frequencies greater than $0.5 \mathrm{~Hz}$ and wavenumbers greater than $1.5 \mathrm{~m}^{-1}$. Cold boils are then identified as contiguous patches of water at least $0.25^{\circ} \mathrm{C}$ cooler than the surrounding water. The candidate boils must also pass the criteria that: 1) they persist at least one second (two frames) in the image data, 2) be at least $10 \mathrm{~cm}$ wide (the approximate resolution of two pixels), and 3) have a combined space-time area less than $250 \mathrm{~m}$ s to be counted as boils. The third criterion ensures removal of any large features associated with very low-frequency apparent temperature variations. Boils identified at each array are characterized by their maximum spanwise ( $y$-direction) diameter and eruption time.

[11] Boil upwelling rate (boils $\min ^{-1}$ ) was tabulated in consecutive six-minute bins at each array as a representative spatial-temporal boil distribution (Figure 2a). At $t \approx 0 \mathrm{~min}$ boils are first detected at the downstream edge of the array. As the boil field develops during decreasing flow depth over the sill the leading edge of the eruption point moves closer to the sill crest until $t \approx 60 \mathrm{~min}$. Boils can still be faintly seen in the thermal imagery data for $t>60 \mathrm{~min}$, but they are not as reliably identified by the automated technique due to the loss of vertical temperature stratification as the cold underlying salt wedge moves out of the region. We focus on $0<t<60$ to test our boil advection model.

[12] The downstream gradient of the boil upwelling rate at each time step was calculated from the discrete $x$-direction (streamwise) difference in the boil upwelling rate and is used to find $L$, the location of the leading edge of upwelling. We define $L$ to be the peak of a Gaussian curve fit to the gradient (Figure $2 \mathrm{~b}$ ) since we expect the leading edge of the boil activity to correspond to the maximum in the gradient. A representative boil diameter (Figure 2c) was calculated as the mean diameter of initial boil eruptions occurring within the region of the leading edge (i.e., from $L-\sigma$ and $L+\sigma$, where $\sigma$ is standard deviation of the fit) to avoid the bias of expanding persistent boils downstream. As $h$ decreased over the ebb, the mean boil diameter in the leading edge increased from about $1 \mathrm{~m}$ to $2 \mathrm{~m}$. Substituting these observations into equation (4), the modeled eruption point $L_{1}$ closely follows observations (Figure 3a), with an rms difference between $L$ and $L_{1}$ of $3.0 \mathrm{~m}$. The robustness of estimate $L_{1}$ is tested by using $L_{2}$ of equation (5), with $U$ defined by the near-surface ADCP velocity, and $U_{z}$ calculated from the average shear in depth-bins located higher than the sill crest. This reduces the rms difference to $1.8 \mathrm{~m}$, though an F-test [Bethea et al., 1995] finds $L_{2}$ is not significantly different from $L_{1}$ to a $95 \%$ confidence level.

\section{Discussion}

[13] A number of assumptions were made to simplify the kinematic boil model. Use of linearly varying velocity profile is supported by qualitative observations of the ADCP velocity profile. Use of a representative advection velocity $U$ appears to be adequate, since equation (5) did not statistically improve predictions of boil eruption distance over equation (4). However, both assumptions may be violated by flow over less obstructing features (e.g., dunes) where the relationship between the shear layer velocities and near surface velocity is not linear [Müller and Gyr, 1986]. In this case, use of equation (4) may result in an over-prediction of $L$.

[14] Representing a 3D vortex loop by a 2D model will necessarily neglect details of the boil development and propagation. Our model assumes instantaneous generation 

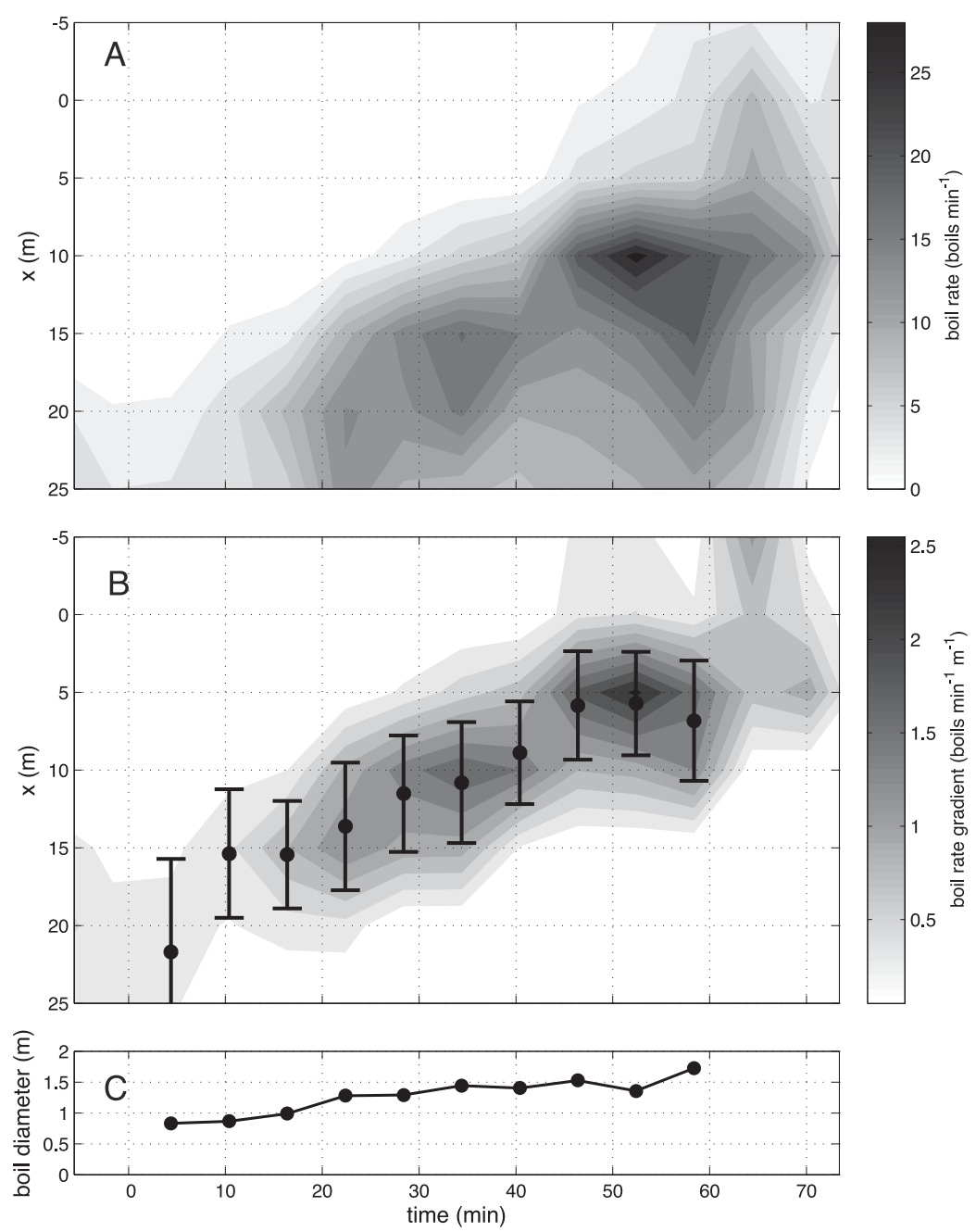

Figure 2. (a) Time-space distribution of observed boil rate downstream of the sill. (b) The maximum of the downstream gradient of the boil activity (shaded) was taken to be the location of the upstream leading edge of boil eruption over time (solid circles, where vertical bars indicate $\pm \sigma$ ). (c) Time history of the mean boil diameter measured within the leading edge of boil upwelling region identified in Figure $2 \mathrm{~b}$.

of full sized boils at the sill crest instead of a smooth growth of the vortex loop as it ascends, implying that the model likely over-predicts vertical boil velocity and under-predict $L$. Moreover, the orientation of the hairpin vortex loop may have angled in the alongstream plane [e.g., Tsai, 1998], so that the vortex pair model would have an alongstream velocity component. This would also lead to systematic overor under-prediction in $L$, depending on the tilt. However, a systematic bias in $L$ is not supported by the results. Using the average observed surface boil diameter as a proxy for $d$ is also a crucial assumption. Substituting fixed boil diameters $d=$ $0.8 \mathrm{~m}$ or $d=1.7 \mathrm{~m}$, the endpoints of the observed range (Figure 2c), into equation (4) results in rms differences with the observations of $6.3 \mathrm{~m}$ and $4.0 \mathrm{~m}$, respectively. By inspection, equation (4) with $d=1.25 \mathrm{~m}$ resulted in the smallest rms difference $(2.0 \mathrm{~m})$.

[15] We further validate our vortex-dipole model by comparing with predictions suggested by laboratory measurements of Müller and Gyr [1986], who captured dye imaged profiles of coherent structures including hairpin vortices that develop in mixing layers, such as the one behind the sill. Their observation of linear growth in the mixed layer wake suggests a simple geometric model for the boil eruption point,

$$
L_{3}=\frac{h}{\alpha}
$$

where $\alpha$ is the angle of mixing layer growth and $L_{3}$ is the extrapolated intersection of the mixing layer with the surface. Values of $\alpha$ determined from Müller and Gyr [1986] range from 0.13 to 0.21 (slopes of $7.5^{\circ}$ to $12^{\circ}$ ). Predictions based on $L_{3}$ approximate the observed eruption locations in the latter half of the focus period, when the flow over the sill is shallowest (Figure 3b), but underpredict the observed downstream locations earlier in the ebb when flow is deeper.

[16] The mismatch of predicted and measured boil eruption distance by equation (6) early in the ebb may be due to two primary factors. First, linear growth of the mixing layer only extends for a limited distance downstream, roughly $2.5 H$, where $H \approx 4 \mathrm{~m}$ is the height of the sill [Deck and Thorigny, 2007; Cherry et al., 1984]. Downstream of this point, there is a flattening in the mixing zone due to 

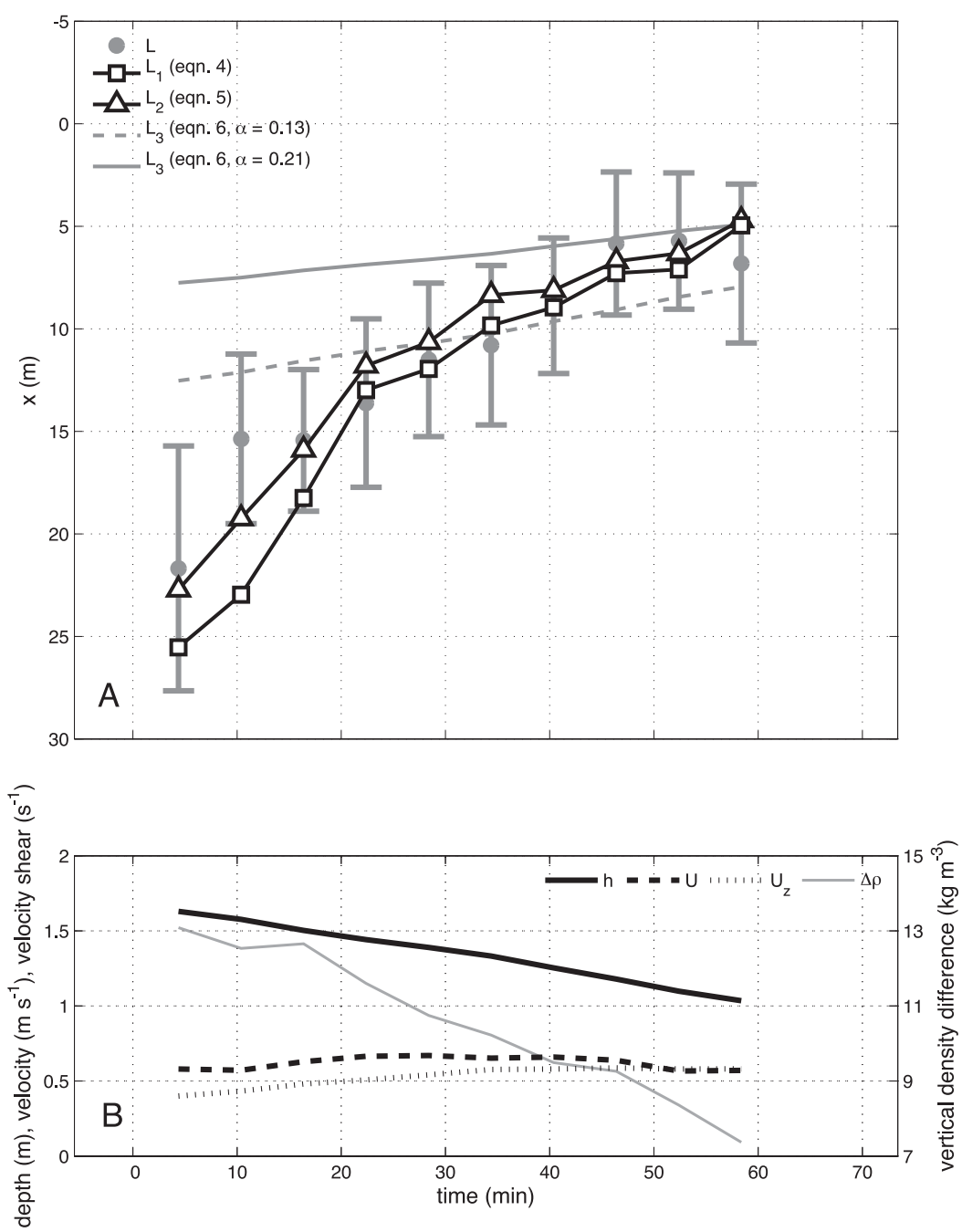

Figure 3. (a) Location of boil eruption point observed (L, solid circles and vertical bars, from Figure $2 \mathrm{c}$ ) and predicted using a vortex-dipole model with linear shear approximation $\left(\mathrm{L}_{1}\right.$, squares $)$, measured velocity shear $\left(\mathrm{L}_{2}\right.$, triangles $)$, and ranges of mixed-layer predictions from Müller and Gyr [1986] ( $\mathrm{L}_{3}$, solid and dashed gray lines). (b) Time series of sill depth $\mathrm{h}$ (bold), vertical density difference $\Delta \rho$, (thin), cross-sill surface velocity $\mathrm{U}$ (dotted) and velocity shear $\mathrm{U}_{\mathrm{z}}$ (dashed).

recirculation. Second, under the stably stratified conditions, evident by the bottom to surface density difference $\Delta \rho$ (Figure 3b), equation (6) is likely to be a lower bound for the surface intersection distance of the mixing layer. Accurate predictions for the downstream expansion of a stratified mixing layer are not as well constrained as for the unstratified case.

[17] The comparisons from the dipole and mixing layer models suggest the following interpretation. Early in the ebb, when the flow is strongly stratified, mixing layer models such as that of Müller and Gyr [1986] significantly over-predict the expansion of the mixing layer, predicting that it will surface well upstream of the observed region of boil activity. During this period, however, energetic boils are generated within the mixing layer that surfaces at a downstream location predicted by the dipole model. Although there is no explicit representation of density stratification in the dipole model, it appears to be robust to stratification. In the development of the dipole model the diameter of the observed surface boil is used to describe the scale of the vortex generated in the shear layer. It appears, therefore, that the effect of stratification is accounted for in the model via the observed boil size; suppression of the scale of vortical structures in the mixing layer during the stratified portion of the ebb results in a smaller observed boil size (Figure 2c). Over the course of the ebb, density stratification decreases and boil diameter increases, despite the fact that the water depth over the sill also decreases. During the weakly stratified period later in the ebb, estimates of boil surfacing distance from the dipole and mixing layer models converge, and both predict the location of boil activity reasonably well.

\section{Conclusions}

[18] Thermal infrared imaging of tidal river flow captured surface eruptions of meter-sized boils generated at a submerged rocky sill. Parameters of the time-varying boil field, including mean boil diameter, boil upwelling rate, and downstream eruption location were quantified from the thermal imagery. The boils are conceptualized as loops of spanwise vorticity (hairpin vortices) generated within the mixing-layer at the sill crest. The resulting boils are hypoth- 
esized to self-propagate to the surface via vortex-pair interaction, modeled as a two-dimensional vortex-dipole. The idealized vortex-dipole model is used to predict the downstream surface eruption location, which in turn depends only on the boil diameter and sill depth. The boil eruption location was accurately predicted in the thermal imagery (rms difference $3.0 \mathrm{~m}$ ) using measured boil diameter and water depth. A test using measured velocity and shear from a collocated ADCP only slightly improved the predictions (rms difference $1.8 \mathrm{~m}$ ) verifying the model to be insensitive to the assumption of linear velocity shear over the sill. Predictions of the boil eruption location based on laboratory measurements of the surface intersection of an internal mixed-layer agree best with observed eruption point when vertical density stratification is low (rms difference $3.7 \mathrm{~m}-6.5 \mathrm{~m}$ ). Density stratification is not explicitly included in the vortex-dipole model, but has no effect on the accuracy of its prediction of boil eruption location.

[19] Acknowledgments. We would like to those who helped with data collection for COHSTREX 2006, especially P. J. Rusello, B. Hayworth, T. Litchendorf, and F. Karig. This research was funded by the Office of Naval Research.

\section{References}

Babakaiff, C. S., and E. J. Hickin (1996), Coherent flow structures in Squamish river estuary, British Columbia, Canada, in Coherent Flow Structures in Open Channels, edited by P. J. Ashworth et al., pp. $321-$ 342, John Wiley, Chichester, U. K.

Batchelor, G. K. (1967), An Introduction to Fluid Dynamics, Cambridge Univ. Press, Cambridge, U. K.

Best, J. (2005a), The fluid dynamics of river dunes: A review and some future research directions, J. Geophys. Res., 110, F04S02, doi:10.1029/ 2004JF000218.

Best, J. (2005b), Kinematics, topology and significance of dune-related macroturbulence: Some observations from the laboratory and field, Spec. Publ. Int. Assoc. Sedimentol., 35, 41-60.
Bethea, R., B. Duran, and T. Boullion (1995), Statistical Methods for Engineers and Scientists, Marcel Dekker, New York.

Cherry, N. J., R. Hillier, and M. E. M. P. Latour (1984), Unsteady measurements in a separated and reattaching flow, J. Fluid Mech., 144, 13-46.

Coleman, J. M. (1969), Brahmaputra River: Channel processes and sedimentation, Sediment. Geol., 3, 129-239.

Deck, S., and P. Thorigny (2007), Unsteadiness of an axisymmetric separating- reattaching flow: Numerical investigation, Phys. Fluids, 19, 065103, doi:10.1063/1.2734996.

Holland, K. T., R. A. Holman, T. C. Lippmann, J. Stanley, and N. Plant (1997), Practical use of video imagery in nearshore oceanographic field studies, IEEE J. Oceanic Eng., 22, 81-92.

Jackson, R. G. (1976), Sedimentological and fluid-dynamic implications of the turbulent bursting phenomenon in geophysical flows, J. Fluid Mech., $77,531-560$.

Kostaschuk, R. A., and M. A. Church (1993), Macroturbulence generated by dunes: Fraser River, Canada, Sediment. Geol., 85, 25-37.

MacDonald, D. G., and A. R. Horner-Devine (2008), Temporal and spatial variability of vertical salt flux in a highly stratified estuary, J. Geophys. Res., 113, C09022, doi:10.1029/2007JC004620.

Matthes, G. M. (1947), Macroturbulence in natural stream flow, Eos Trans. $A G U, 28,255-265$.

Müller, A., and A. Gyr (1986), On the vortex formation in the mixing layer behind dunes, J. Hydraul. Res., 24, 359-375.

Nezu, I., and H. Nakagawa (1993), Turbulence in Open-Channel Flows, 281 pp., Balkema, Rotterdam, Netherlands.

Tsai, W.-T. (1998), A numerical study of the evolution and structure of a turbulent shear layer under a free surface, J. Fluid Mech., 354, 239-276.

Venditti, J. G., and B. O. Bauer (2005), Turbulent flow over a dune: Green River, Colorado, Earth Surf. Processes Landforms, 30, 289-304.

Yue, W., C.-L. Lin, and V. C. Patel (2005), Coherent structures in openchannel flows over a fixed dune, J. Fluids Eng., 127, 858-864.

Yue, W., C.-L. Lin, and V. C. Patel (2006), Large-eddy simulation of turbulent flow over a fixed two-dimensional dune, J. Hydraul. Eng., $132,643-651$.

C. C. Chickadel and A. T. Jessup, Applied Physics Laboratory, University of Washington, 1013 NE 40th Street, Box 355640, Seattle, WA 98105, USA. (chickadel@apl.washington.edu)

A. R. Horner-Devine and S. A. Talke, Civil and Environmental Engineering, University of Washington, 201 More Hall, Box 352700, Seattle, WA 98105 , USA. 